\title{
Varietal Reactions of Turmeric towards Leaf Spot and Rhizome Rot Disease in Konkan Region of Maharashtra, India
}

\author{
R. R. Rathod $^{1^{*}}$, A. P. Suryawanshi ${ }^{2}$, R. G. Khandekar ${ }^{3}$, \\ U. B. Pethe ${ }^{4}$ and C. B. Gondhalekar ${ }^{1}$ \\ ${ }^{1}$ Department of Plant Pathology, College of Agriculture, Dapoli, India \\ ${ }^{2}$ Department of Plant Pathology, ${ }^{3}$ Department of Horticulture, ${ }^{4}$ Department of Agriculture \\ Botany, Dr. B. S. Konkan Krishi Vidyapeeth, Dapoli, India \\ *Corresponding author
}

\section{A B S T R A C T}

\section{Keywords}

Curcuma longa,

Screening,

Susceptible,

Resistant,

Moderately

Resistant

Article Info

Accepted:

12 September 2019

Available Online:

10 October 2019
Turmeric (Curcuma longa L.) is well known for its medicinal value, its cultivation is hindered by several diseases viz., leaf spot, anthracnose and rhizome rot. These diseases are managed by using the fungicides, which leads to development of resistant strains besides the environmental pollution and also the residue problem on final produce. Host resistance seems to be most promising means of disease management. Therefore, field screening trial was planned and conductd in Konkan region of Maharashtra at Asond Block, AICRP on Spices, DBSKKV, Dapoli for three years (2015-16, 2016-17 \& 2017-18) to identify the disease resistant sources against Colletotrichum leaf spot and Pythium rhizome rot with high yield potential. Three years average data revealed that, out of 30 turmeric varieties screened, 29 were found moderately resistant (MR) and only one variety Kanti was susceptible (S) to Colletotrichum leaf spot. But, on the yield basis amongst all varieties, four varieties viz., Krishna, Sudharsana, Narendra Haldi and Pratibha performed well. Minimum leaf spot intensity was recorded in Pratibha (11.69\%), Sudharsana (12.99\%), Krishna (13.23\%) and NarendraHaldi (13.82\%) with high yield of $17.66 \mathrm{~kg} / \mathrm{plot}$, $18.86 \mathrm{~kg} / \mathrm{plot}, 19.46 \mathrm{~kg} / \mathrm{plot}$ and $18.10 \mathrm{~kg} / \mathrm{plot}$, respectively. Whereas, for rhizome rot, the varieties Pratibha,Sudharsana and NarendraHaldi were tolerant $(\mathrm{T})$ and only one variety Krishna was found moderately resistant (MR). It is concluded from present study that, Krishna, Sudharsana, Narendra Haldi and Pratibha, which may be recommended to farmers for cultivation in Konkan region of Maharashtra.

\section{Introduction}

Haldi or turmeric (Curcum alonga L.) is one of the most important condiment and colouring agents of the world. Turmeric is also a commodity of auspicious religious ceremonies in India. It is being used essentially in curry powder and is a common food preservative. Turmeric is grown in the states of Tamil Nadu, Kerala, Assam, Andhra 
Pradesh, West Bengal and Maharashtra. Turmeric belongs to family Zingiberaceae.

It is originated from Tropical South Asia. It is named as "Indian saffron". Its active ingredient is curcumin. Indian turmeric is considered the best in the world due to presence of high curcumin content. Turmeric contains essential oils up to $5 \%$ and curcumin up to 5\%, a polyphenol (Anonymous 2012).

Though it is well known for its medicinal value, its cultivation is hindered by several diseases. Turmeric is susceptible to diseases viz., leaf spot, anthracnose and rhizome rot. Leaf spot caused by Colletotrichum capsici, was found increasing and occurring regularly every year. It has become as major constraint in successful cultivation of turmeric.

The disease resulted in drastic reduction in rhizome yield. Rhizome rot caused by Pythium sp. is a major constraint in all turmeric-growing areas of India (Rathiah, 1987; Nageshwar Rao, 1994; Ramarethinam and Rajagopal, 1999).

Different species of Pythium is involved in causing rhizome rot in different parts of the country. Rhizomerot of turmeric incited by Pythium aphanidermatum was first reported in Sri Lanka by Park (1934), later it was reported as $P$. graminicoloumfrom the Krishna district of Andhra Pradesh by Ramakrishnan and Sowmini (1954) and P. myriotylum from Assam by Rathiah (1982).

At present the disease was managed by using the fungicides which lead to development of resistant strains besides the environmental pollution and also the residue problem on final produce. Hence, field screening trial was conducted during three years (2015-16, 2016$17 \& 2017-18)$ to identify the disease resistant varieties in the available thirty different varieties against the Colletotrichum leaf spot and Pythium rhizome rot. Use of resistant varieties is the most economic and easily adoptable method in integrated disease management. Identification of high yielding turmeric varieties which are relatively resistant or tolerant to major diseases are of utmost importance for increasing the production and productivity of turmeric in konkan region of Maharashtra.

\section{Materials and Methods}

Thirty different varieties of turmeric viz., 1) Krishna, 2) Rajpuri, 3) Salem local, 4) Ranga, 5) Pratibha, 6) Roma, 7) Pant Peethabh, 8) Narendra Haldi, 9) Sona, 10) BSR-1, 11) BSR-2, 12) Kedaram, 13) Panjab Haldi-1, 14) Panjab Haldi-2, 15) Sobha, 16) Prabha, 17) Suvarna, 18) Suguna, 19) Sudharshna, 20) Kanti, 21) Sikandarabad, 22) Alleppy Supreme, 23) SB - 10843, 24) RH - 5, 25) Arunachal Local, 26) Cochbet, 27) Tekurpeta, 28) Jalpalguri local, 29) Allam puram and 30) Suranjana, were planted at 37.5 $\mathrm{X} 30 \mathrm{~cm}$ spacing at Asond Farm, AICRP on Spices, Dapoli (Dr. B. S. Konkan Krishi Vodyapeeth, Dapoli) during the period of 2015 -16 to 2017-18.

Observations were recorded on Per cent Disease Intensity (1-5 point scale), Per cent Disease Incidence (1-6 point scale). Disease score chart for leaf spot disease and rhizome rot disease are as follows -

Disease Score Chart (leaf spot disease)

$\begin{array}{lll}\text { Grade } & \begin{array}{l}\text { Incidence } \\ (\%)\end{array} & \text { Category } \\ 1 & \text { Upto 5\% } & \text { Highly Resistant } \\ 2 & 5-10 \% & \text { Resistant } \\ 3 & 11-25 \% & \begin{array}{l}\text { Moderately } \\ \text { Resistant }\end{array} \\ 4 & 26-50 \% & \begin{array}{l}\text { Susceptible } \\ \text { Highly Susceptible }\end{array} \\ 5 & >50 \% & \end{array}$


Disease Score Chart (Rhizome rot disease)

$\begin{array}{ll}\text { Grade } & \text { Incidence }(\%) \\ 1 & 0.0 \\ 2 & 1.0-5.0 \\ & \\ 3 & 6.0-15.0 \\ 4 & 16.0-25.0 \\ & \\ 5 & 26.0-40.0 \\ 6 & \text { Above } 40.0\end{array}$

Results and Discussion

The response of the verities against

Resistant Moderately

Resistant

Tolerant

Moderately

Tolerant

Susceptible

Highly

Susceptible
Colletotrichum leaf spot are given in Table 1. The results revealed that, out of thirty varieties of turmeric screened, twenty nine varieties were observed moderately resistant.

The severity of the leaf spot showed variation among the turmeric verities screened in the range of 11.69 to 24.86 PDI. The interest point observed on foliar diseases in the present study is that, no other leaf spot/ blight/ blotch diseases were observed on these varieties.

In present study, only one variety, Kanti was found susceptible (30.74 PDI) against leaf spot disease of turmeric.

Table.1 Reactions of turmeric against Colletotrichum leaf spot disease of turmeric

\begin{tabular}{|c|c|c|c|c|c|c|c|}
\hline $\begin{array}{c}\text { Sr. } \\
\text { No }\end{array}$ & Variety & $\begin{array}{c}\text { Per cent } \\
\text { Disease } \\
\text { Intensity }\end{array}$ & Reactions & $\begin{array}{c}\text { Sr. } \\
\text { No }\end{array}$ & Variety & $\begin{array}{c}\text { Per cent } \\
\text { Disease } \\
\text { Intensity }\end{array}$ & Reactions \\
\hline $\mathbf{1 .}$ & Krishna & 13.23 & MR & 16. & Prabha & 19.53 & MR \\
\hline $\mathbf{2 .}$ & Rajpuri & 16.21 & MR & 17. & Suvarna & 13.09 & MR \\
\hline $\mathbf{3 .}$ & Salem Local & 21.12 & MR & 18. & Suguna & 13.01 & MR \\
\hline $\mathbf{4 .}$ & Ranga & 20.59 & MR & 19. & Sudarsana & 12.99 & MR \\
\hline $\mathbf{5 .}$ & Pratibha & 11.69 & MR & 20. & Kanti & 30.74 & S \\
\hline $\mathbf{6 .}$ & Roma & 20.07 & MR & 21. & Sikandarabad & 21.51 & MR \\
\hline $\mathbf{7 .}$ & Pant Peetabh & 16.10 & MR & 22. & Alleppeysupreme & 24.86 & MR \\
\hline $\mathbf{8 .}$ & NarendraHaldi & 13.82 & MR & 23. & SB-10843 & 18.49 & MR \\
\hline $\mathbf{9 .}$ & Sona & 12.24 & MR & 24. & RH-5 & 18.32 & MR \\
\hline $\mathbf{1 0 .}$ & BSR-1 & 15.13 & MR & 25. & Arunachal Local & 22.38 & MR \\
\hline $\mathbf{1 1 .}$ & BSR-2 & 22.80 & MR & 26. & Cochbet & 16.49 & MR \\
\hline $\mathbf{1 2 .}$ & Kedaram & 16.72 & MR & 27. & Tekurpeta & 19.61 & MR \\
\hline $\mathbf{1 3 .}$ & Punjab Haldi-1 & 16.30 & MR & 28. & Jalpalguri Local & 19.34 & MR \\
\hline $\mathbf{1 4 .}$ & Punjab Haldi-2 & 15.99 & MR & 29. & Allampuram & 18.69 & MR \\
\hline 15. & Sobha & 15.90 & MR & 30. & Suranjana & 19.46 & MR \\
\hline & & & & & & & \\
\hline
\end{tabular}


Table.2 Reactions of turmeric against Pythium rhizome rot of turmeric

\begin{tabular}{|c|c|c|c|c|c|c|c|}
\hline $\begin{array}{c}\text { Sr. } \\
\text { No. }\end{array}$ & Variety & $\begin{array}{c}\text { Per cent } \\
\text { Disease } \\
\text { Incidence }\end{array}$ & Reactions & $\begin{array}{c}\text { Sr. } \\
\text { No. }\end{array}$ & Variety & $\begin{array}{c}\text { Per cent } \\
\text { Disease } \\
\text { Incidence }\end{array}$ & Reactions \\
\hline $\mathbf{1 .}$ & Krishna & $\mathbf{4 . 9 2}$ & MR & 16. & Prabha & 8.86 & $\mathrm{~T}$ \\
\hline $\mathbf{2 .}$ & Rajpuri & 11.55 & $\mathrm{~T}$ & 17. & Suvarna & 7.58 & $\mathrm{~T}$ \\
\hline $\mathbf{3 .}$ & Salem Local & 12.98 & $\mathrm{~T}$ & 18. & Suguna & 7.25 & $\mathrm{~T}$ \\
\hline $\mathbf{4 .}$ & Ranga & 11.62 & $\mathrm{~T}$ & 19. & Sudarsana & 9.58 & $\mathrm{~T}$ \\
\hline $\mathbf{5 .}$ & Pratibha & 11.07 & $\mathrm{~T}$ & 20. & Kanti & 5.40 & $\mathrm{~T}$ \\
\hline $\mathbf{6 .}$ & Roma & 12.08 & $\mathrm{~T}$ & 21. & Sikandarabad & 7.80 & $\mathrm{~T}$ \\
\hline $\mathbf{7 .}$ & Pant Peetabh & 10.05 & $\mathrm{~T}$ & 22. & Alleppey supreme & 9.99 & $\mathrm{~T}$ \\
\hline $\mathbf{8 .}$ & NarendraHaldi & 8.19 & $\mathrm{~T}$ & 23. & SB-10843 & 10.79 & $\mathrm{~T}$ \\
\hline $\mathbf{9 .}$ & Sona & 9.89 & $\mathrm{~T}$ & 24. & RH-5 & 8.28 & $\mathrm{~T}$ \\
\hline $\mathbf{1 0 .}$ & BSR-1 & 8.61 & $\mathrm{~T}$ & 25. & Arunachal Local & 8.70 & $\mathrm{~T}$ \\
\hline $\mathbf{1 1 .}$ & BSR-2 & 9.73 & $\mathrm{~T}$ & 26. & Cochbet & 10.68 & $\mathrm{~T}$ \\
\hline $\mathbf{1 2 .}$ & Kedaram & 8.83 & $\mathrm{~T}$ & 27. & Tekurpeta & 9.82 & $\mathrm{~T}$ \\
\hline $\mathbf{1 3 .}$ & Punjab Haldi-1 & 9.55 & $\mathrm{~T}$ & 28. & Jalpalguri Local & 10.68 & $\mathrm{~T}$ \\
\hline $\mathbf{1 4}$ & Punjab Haldi-2 & 9.84 & $\mathrm{~T}$ & 29. & Allampuram & 8.86 & $\mathrm{~T}$ \\
\hline $\mathbf{1 5}$ & Sobha & 11.61 & $\mathrm{~T}$ & 30. & Suranjana & 7.58 & $\mathrm{~T}$ \\
\hline
\end{tabular}

Table.3 Average yield performance of turmeric varieties

\begin{tabular}{|c|c|c|c|c|c|c|c|}
\hline \multirow{2}{*}{$\begin{array}{l}\text { Sr. } \\
\text { No. }\end{array}$} & \multirow[t]{2}{*}{ Variety } & \multicolumn{2}{|c|}{ Average Yield } & \multirow{2}{*}{$\begin{array}{l}\text { Sr. } \\
\text { No. }\end{array}$} & \multirow[t]{2}{*}{ Variety } & \multicolumn{2}{|c|}{ Average Yield } \\
\hline & & kg/ plot & t/ha & & & kg/plot & t/ha \\
\hline 1. & Krishna & 19.46 & 31.14 & 16. & Prabha & 13.36 & 21.37 \\
\hline 2. & Rajpuri & 12.24 & 19.58 & 17. & Suvarna & 12.77 & 20.44 \\
\hline 3. & Salem Local & 11.70 & 18.72 & 18. & Suguna & 12.86 & 20.58 \\
\hline 4. & Ranga & 12.77 & 20.44 & 19. & Sudarsana & 18.86 & 30.18 \\
\hline 5. & Pratibha & 17.66 & 28.26 & 20. & Kanti & 11.20 & 17.93 \\
\hline 6. & Roma & 13.27 & 21.24 & 21. & Sikandarabad & 11.88 & 19.01 \\
\hline 7. & Pant Peetabh & 12.38 & 19.82 & 22. & Alleppeysupreme & 15.23 & 24.37 \\
\hline 8. & NarendraHaldi & 18.10 & 28.97 & 23. & SB-10843 & 10.60 & 16.96 \\
\hline 9. & Sona & 13.47 & 21.56 & 24. & RH-5 & 12.86 & 20.58 \\
\hline 10. & BSR-1 & 11.97 & 19.15 & 25. & Arunachal Local & 10.40 & 16.65 \\
\hline 11. & BSR-2 & 13.06 & 20.90 & 26. & Cochbet & 10.37 & 16.60 \\
\hline 12. & Kedaram & 13.64 & 21.83 & 27. & Tekurpeta & 10.78 & 17.24 \\
\hline 13. & Punjab Haldi-1 & 12.70 & 20.33 & 28. & Jalpalguri Local & 9.67 & 15.46 \\
\hline 14. & Punjab Haldi-2 & 11.74 & 18.78 & 29. & Allampuram & 14.58 & 23.33 \\
\hline 15. & Sobha & 11.80 & 18.88 & 30. & Suranjana & 12.20 & 19.53 \\
\hline
\end{tabular}


Table.4 Economics of turmeric

\begin{tabular}{|c|c|c|c|c|c|c|}
\hline $\begin{array}{l}\text { Sr. } \\
\text { No. }\end{array}$ & Treatment & $\begin{array}{l}\text { Per cent } \\
\text { Disease } \\
\text { Incidence } \\
\text { (PDI) }\end{array}$ & Yield (t/ha) & $\begin{array}{c}\text { Gross value } \\
\text { of yield } \\
\text { (Rs./ha) }\end{array}$ & $\begin{array}{c}\text { Total } \\
\text { cost } \\
\text { (Rs./ha) }\end{array}$ & $\begin{array}{c}\text { Cost: } \\
\text { Benefit } \\
\text { ratio }\end{array}$ \\
\hline 1 & 2 & 3 & 4 & 6 & 7 & 8 \\
\hline 1 & Krishna & 13.23 & 31.14 & 1245733.33 & 473042 & $1: 2.63$ \\
\hline 2 & Rajpuri & 16.21 & 19.58 & 783200.00 & 473042 & $1: 1.66$ \\
\hline 3 & Salem Local & 21.12 & 18.72 & 748666.67 & 473042 & $1: 1.58$ \\
\hline 4 & Ranga & 20.59 & 20.44 & 817600.00 & 473042 & $1: 1.73$ \\
\hline 5 & Pratibha & 11.69 & 28.26 & 1130266.67 & 473042 & $1: 2.39$ \\
\hline 6 & Roma & 20.07 & 21.24 & 849466.67 & 473042 & $1: 1.80$ \\
\hline 7 & Pant Peetabh & 16.10 & 19.82 & 792666.67 & 473042 & $1: 1.68$ \\
\hline 8 & NarendraHaldi & 13.82 & 28.97 & 1158666.67 & 473042 & $1: 2.45$ \\
\hline 9 & Sona & 12.24 & 21.56 & 862266.67 & 473042 & $1: 1.82$ \\
\hline 10 & BSR-1 & 15.13 & 19.15 & 766133.33 & 473042 & $1: 1.62$ \\
\hline 11 & BSR-2 & 22.80 & 20.90 & 835866.67 & 473042 & $1: 1.77$ \\
\hline 12 & Kedaram & 16.72 & 21.83 & 873066.67 & 473042 & $1: 1.85$ \\
\hline 13 & Punjab Haldi-1 & 16.30 & 20.33 & 813066.67 & 473042 & $1: 1.72$ \\
\hline 14 & Punjab Haldi-2 & 15.99 & 18.78 & 751066.67 & 473042 & $1: 1.59$ \\
\hline 15 & Sobha & 15.90 & 18.88 & 755200.00 & 473042 & $1: 1.60$ \\
\hline 16 & Prabha & 19.53 & 21.37 & 854933.33 & 473042 & $1: 1.81$ \\
\hline 17 & Suvarna & 13.09 & 20.44 & 817466.67 & 473042 & $1: 1.73$ \\
\hline 18 & Saguna & 13.01 & 20.58 & 823066.67 & 473042 & $1: 1.74$ \\
\hline 19 & Sudarsana & 12.99 & 30.18 & 1207066.67 & 473042 & $1: 2.55$ \\
\hline 20 & Kanti & 30.74 & 17.93 & 717066.67 & 473042 & $1: 1.52$ \\
\hline 21 & Sikandarabad & 21.51 & 19.01 & 760266.67 & 473042 & $1: 1.61$ \\
\hline 22 & Alleppey Supreme & 24.86 & 24.37 & 974666.67 & 473042 & $1: 2.06$ \\
\hline 23 & SB-10843 & 18.49 & 16.96 & 678400.00 & 473042 & $1: 1.43$ \\
\hline 24 & RH-5 & 18.32 & 20.58 & 823200.00 & 473042 & $1: 1.74$ \\
\hline 25 & Arunachal Local & 22.38 & 16.65 & 665866.67 & 473042 & $1: 1.41$ \\
\hline 26 & Cochbet & 16.49 & 16.60 & 664133.33 & 473042 & $1: 1.40$ \\
\hline 27 & Tekurpeta & 19.61 & 17.24 & 689733.33 & 473042 & $1: 1.46$ \\
\hline 28 & Jalpalguri Local & 19.34 & 15.46 & 618533.33 & 473042 & $1: 1.31$ \\
\hline 29 & Allampuram & 18.69 & 23.33 & 933200.00 & 473042 & $1: 1.97$ \\
\hline 30 & Suranjana & 19.46 & 19.53 & 781066.67 & 473042 & $1: 1.65$ \\
\hline
\end{tabular}


This study is in accordance with the finding of Mallikarjun (1996), Phlip, J. and Nair, P.C. (1981), Gorawar et al., (2006), Singh (2007) and Jagtap et al., (2013).

In turmeric, rhizome rot is another destructive disease that also causes economic damage to the crop. A total of 30 varieties were screened against Pythium rhizome rot of turmeric, out of which, only one, Krishna variety was found moderately resistant (4.92 PDI) and rest all twenty nine varieties were tolerant (Table 2). On the yield basis all varieties performed well but on basis of per cent disease intensity incidence of yield the varieties viz., Krishna (31.14 t/ha), Sudharsana (30.18 t/ha), Narendra Haldi (28.97 t/ha) and Pratibha (28.26 t/ha) were found superior over other varieties under study (Table 3).

Table 4, revealed that, highest $\mathrm{C}: \mathrm{B}$ ratio was recorded in Krishna, Sudharsana, Narendra Haldi and Pratibhaas 1: 2.63, 1: 2.55, 1: 2.45 and 1: 2.39 respectively.

On the yield basis all varieties performed well but on basis of percent disease intensity incidence and yield of the varieties viz., Krishna, Sudharsana, Narendra Haldi and Pratibha were found superior over other varieties under study, which may be recommended to farmers for cultivation in konkan region of Maharashtra.

Out of thirty varieties screened, it is recommended that four varieties viz., Krishna, Sudharsana, Narendra Haldi and Pratibhaare moderately resistant to Colletotrichum leaf spot and Pythium rhizome rot with good yield.

\section{Acknowledgement}

Dr. R. R. Rathod, is grateful to the Ex-Head, Dept. of Pl Pathology (Dr. V.S. Pande), Head, Department of Horticulture (Dr. B. R. Salvi) for the help provided. He wish to express sincere gratitude), The Associate Dean, COA, Dapoli (Dr. U.V. Mahadkar) for their guidance. The support rendered by the Director of Research, DBSKKV, Dapoli (Dr. P.M. Haldankar), the Dean, DBSKKV, Dapoli (Dr. S.S. Narkhede), The Director of Extension Education, DBSKKV, Dapoli (Dr. S. G. Bhave) and the Director, IISR, Kozhikode (Dr. K. NirmalBabu) are sincerely acknowledged.

\section{References}

Gorawar Mamatha M., Hegde, Y. R. and Kulkarni S. 2006. Screening of genotypes and effect of fungicides against leaf blight of turmeric.

Jagtap, G. P., Mali, A. K. and Utpal Dey 2013. Bioeficacy of fungicides, bio-control agents and botanicals against leaf spot of turmeric incited by Colletotrichum capsici.African Journal of Microbiology Research. Vol. No. 7 (18): pp 1865-1873.

Mallikarjun, G. 1996. Studies on Alternaria alternata, M.Sc. (Agri) Thesis, University of Agricultural Sciences, Dharwad. pp-48

NageshwarRao T.G. 1994. Turmeric rhizome rot and its management. Spice India.7: 17-19.

Narsimha Rao, S.. Ravindra Kumar, K. and Prameela, M. 2015. In vivo screening of Turmeric (Curcuma longa L.) germplasm of different duration groups against Leaf spot caused by Colletotrichum capsici ((Sydner) Butl \& Bis).

Ramakrishanan, T.S. and Sowmini, C. K. 1954. Rhizome root rot of turmeric caused by $\mathrm{P}$. graminicolum. Ind. Phytopath. 7: 152-159.

Ramana K.V. and Eapen, S. J. 1995. Parasitic nematodes and their management in major spices. J. of Spices and Aromatic Crops.4(1): 1-16 
Ramarethianum, C. and Rajagopal B. 1999. Efficacy of Trichoderma spp. organic amendments and seed dressing fungicides on rhizome rot of turmeric. Pestology. 13: 21-30.

Rathaiah Y. 1982. Rhizome rot of turmeric. Indian Phytopath.35: 415 -417.
Rethiah Y. 1987. Control of soft rot of ginger with Ridomil. Pesticides. 21: 29-30.

Singh, A. K. 2007. Screening of turmeric cultivars against Taphrina and Colletotrichum leaf spot diseases. Ind. J. of Pl. Prot. 35 (1): 142-143.

\section{How to cite this article:}

Rathod, R. R., A. P. Suryawanshi, R. G. Khandekar, U. B. Pethe and Gondhalekar, C. B. 2019. Varietal Reactions of Turmeric towards Leaf Spot and Rhizome Rot Disease in Konkan Region of Maharashtra, India. Int.J.Curr.Microbiol.App.Sci. 8(10): 1735-1741.

doi: https://doi.org/10.20546/ijcmas.2019.810.202 